\title{
La tensión redistribución-reconocimiento en las políticas sociales: notas y reflexiones desde la teoría política feminista
}

\section{The redistribution-recognition tension in social policies: notes and reflections from feminist political theory}

SuYai MaLEn García GuALDA

IPEHCS-CONICET-UNCO

Recibido: $28 / 5 / 2020$

Aceptado: 8/9/2020

doi: https://doi.org/10.20318/femeris.2021.5929

\begin{abstract}
Resumen. En este trabajo realizamos un sucinto recorrido por el dilema redistribuciónreconocimiento desde los aportes de la filosofía y teoría política contemporánea, con especial atención en la perspectiva de la feminista Nancy Fraser. La autora entiende que la justicia requiere de la articulación entre redistribución y reconocimiento, ya que de forma aislada ambas dimensiones resultan insuficientes y, por esto, considera que pensar en una dicotomía es sostener una falsa antítesis que nubla toda posibilidad de comprender y transformar la compleja realidad contemporánea. Partimos de entender que la dimensión de género nos coloca frente a la tensión que existe entre reconocimiento y redistribución, puesto que pone en evidencia la imperiosa necesidad de articular la igualdad social con el reconocimiento de la diferencia a fin de garantizar la construcción de una sociedad justa y equitativa. A la luz del citado dilema exponemos el desarrollo de nuestro incipiente análisis de dos políticas sociales que han marcado significativamente los últimos años en Argentina: la Asignación Universal por Hijo y el Programa Ellas Hacen (modificado por la anterior gestión de gobierno). A nuestro juicio, ambas experiencias aportan interesantes datos para reflexionar sobre la urgencia de transversalizar la perspectiva interseccional de género en las políticas de estado.

Palabras clave: reconocimiento, redistribución, perspectiva de género, políticas sociales, justicia social.
\end{abstract}

Abstract. In this work, we make a brief overview of the redistribution-recognition dilemma from the contemporary political theory and philosophy, with special attention to the perspective of the feminist Nancy Fraser. The author understands that justice requires the articulation between redistribution and recognition, since in isolation both dimensions are insufficient, she considers that to think of a dichotomy is to sustain a false antithesis that blurs all possibility of understanding and transforming the reality. We start by understanding that the gender dimension places us in front of the tension that exists between recognition and redistribution. This highlights the urgent need to articulate social equality with the recognition of the difference to guarantee the construction of a just and equitable society. Considering this

*gsuyai@hotmail.com 
dilemma, we present the development of our incipient analysis of two social policies that have significantly marked the last years in Argentina: the AUH and "Ellas Hacen" (modified by the previous administration). In our opinion, both experiences provide interesting data that reflect on the urgency of mainstreaming the intersectional perspective of gender in state policies.

Keywords: recognition, redistribution, gender perspective, social policies, social justice.

\section{Introducción}

Es indudable que la publicación de la obra Teoría de la Justicia (2000 [1971]) de John Rawls, en la década de los setenta, inauguró una etapa nutrida de debates sobre los principios que deben sustentar las instituciones de una sociedad justa. A partir de ese momento, la "justicia social" cobró centralidad como uno de los pilares de la reflexión y acción filosófico-política. Dentro de estos debates podemos identificar, por un lado a los/as defensores de la justicia distributiva (el igualitarismo liberal, el igualitarismo de la suerte y algunas formas de socialismo vinculadas a la escuela del marxismo analítico) y, también, a las corrientes que conforman el denominado "comunitarismo". Y, por otro lado, ubicamos a los/as pensadores/as afines al libertarismo de derecha, cuyo principal exponente es Robert Nozick. Cabe señalar que dicho autor rechaza la justicia distributiva como categoría válida y propone una justicia retributiva de libre mercado.

Entre los/as autores/as que han abordado la noción de justicia distributiva, a partir de un enfoque de derechos, se encuentra Amartya Sen. Para este pensador la métrica igualitaria escogida por Rawls, la igualdad de "bienes primarios"1", es altamente imperfecta porque se concentra en ciertos bienes "objetivos" y descuida el modo diferente en que los mismos pueden impactar sobre distintos individuos, quienes viven en contextos disímiles (Gargarella, 1999). Según algunos/as estudiosos/as, Amartya Sen ha logrado establecer un punto intermedio entre el subjetivismo utilitario y el objetivismo de los bienes primarios, proponiéndose igualar a las personas en sus capacidades básicas². Para este teórico hay injusticias remediables, por ello se propone razonar operativamente sobre ellas. En ese sentido, Roxana Mazzola (2015) afirma que para Sen el núcleo de las políticas sociales ha de ser la redistribución o distribución secundaria del ingreso con la finalidad de aportar a la construcción de una sociedad justa.

Las políticas sociales son parte constitutiva del sistema de políticas públicas considerándolas como programas de acción de una autoridad política (Meny y Thoenig en Mazzola, 2015, p. 7). En consecuencia, las políticas sociales pueden ser definidas como la toma

\footnotetext{
${ }^{1}$ John Rawls entiende que los/as sujetos/as persiguen o buscan de manera racional ciertos bienes que les permiten desempeñarse socialmente (Gallego, 2016, p. 24). Estos "bienes primarios" son derechos y libertades, los poderes y las oportunidades vinculadas a posiciones sociales, así como la riqueza e ingresos y el auto-respeto o el sentido del propio valer (Rawls, 2000, p. 95).

${ }^{2}$ Amartya Sen entiende a las capacidades como un conjunto de funcionamientos valiosos. La métrica de las capacidades, dice Gallego (2016), se concentra no en los bienes en sí mismos sino que en lo que los bienes les permiten hacer a los agentes que disponen de ellos. Es decir, esta métrica pretende observar la diversidad de circunstancias que dan lugar a la desigualdad. Así, una capacidad "supone la posibilidad de alcanzar estados y opciones de acción”, los cuales son denominados "funcionalidades" y se consideran constitutivos de las personas (Gallego, 2016, p. 27).
} 
de postura del Estado a través de la que expresa una idea de justicia distributiva sobre cierta cuestión social, por ejemplo la distribución de renta para garantizar los derechos de las infancias u otros sectores vulnerables o desaventajados. De modo que, desde esta perspectiva, las políticas sociales refieren a la intervención del Estado a fin de atenuar o hacer desaparecer las desigualdades por medio de la designación (eficiente de recursos), distribución (equitativa de la renta) y estabilidad (económica). Todo esto en busca de la equidad y con el fin último de construir y consolidar ciudadanía. Es por esto que surge la necesidad de abordar las políticas sociales desde una mirada finalística, sin perder de vista el carácter conflictivo y relacional de las mismas (Mazzola, 2015).

En este sentido, los autores Adelantado, Noguera y Rambla (2000) proponen pensar la vinculación o relación recíproca que existe entre las políticas sociales y la estructura social. Destacan tres grandes corrientes que han intentado explicar las políticas sociales en el período de posguerra: el neo-marxismo, la(s) tradición(es) no marxistas y la propuesta de Esping-Andersen (2000). Esta última parte de entender que "el surgimiento de distintos modelos de Estado de Bienestar se explica en base a diferentes sistemas de estratificación social, que dan lugar a diferentes alianzas de clases" y que "la política social no sólo compensa o reduce las desigualdades originadas en otros ámbitos, sino que también puede reproducirlas, aumentarlas e incluso constituirlas como tales" (Adelantado et al, 2000, p. 3). Esping-Andersen (2000) considera que las funciones fundamentales del Estado de Bienestar son: desmercantilizar, redistribuir y desfamiliarizar. Así, como dijimos, Adelantado et al (2000) proponen pensar la relación entre la estructura social y las políticas sociales, y para ello definen a la estructura social como una configuración de instituciones, reglas y recursos que atribuyen condiciones de vida desiguales a las personas en cierto momento y lugar. En esta concepción de estructura pueden identificarse tres dimensiones: esfera de acción, ejes de desigualdad y actores colectivos. Y es en este punto en el que cobran vital relevancia las contribuciones de autores/as denominados/as comunitaristas.

Fue a partir de la década de los ochenta que emergieron con fuerza los/as autores/as comunitaristas, entre ellos/as M. Walzer (1993) quien aportó su mirada sobre las "esferas de la justicia". Las teorías comunitaristas no liberales incorporaron el problema (re)distributivo en un marco cultural más amplio, en función de las significaciones, sentidos e identificaciones que se asignan a los bienes sociales. En el comunitarismo, una distribución puede ser pautada o no, procedimental o de resultado final, en función de la cultura de la que se trate. Los bienes primarios rawlsianos, los recursos dworkinianos ${ }^{3}$, o el bienestar utilitarista son reemplazados por los bienes sociales en tanto significaciones. Y el reconocimiento de las identidades y particularidades culturales, que en las teorías igualitarias universalistas recibían poca atención, adquieren notable relevancia. De este modo aparece la cuestión del reconocimiento como una dimensión central para pensar una sociedad justa.

\footnotetext{
${ }^{3}$ Para Dworkin, la igualdad de recursos es un mecanismo de distribución igualitaria de los recursos socioeconómicos. En este sentido, como explica Gallego (2016), Dworkin “favorece una igualdad de recursos, siempre y cuando se introduzca como correctivo un principio de responsabilidad por las elecciones personales que permita distinguir quiénes deben beneficiarse del esquema redistributivo y quiénes no" (p. 25).
} 
En las páginas siguientes iniciamos un breve recorrido por el dilema redistribuciónreconocimiento desde los aportes de la filosofía y teoría política contemporánea, con especial atención en la perspectiva de la autora feminista Nancy Fraser. Para esta pensadora, gran parte de las teorías feministas han desplazado a la cuestión de género desde las teorías cuasi-marxistas hacia las post-marxistas, centradas en lo cultural e identitario. De hecho, su planteo se centra en advertir cómo en la etapa postsocialista (cuando se hicieron evidentes los límites de los modelos de bienestar y soviético y emergió con ímpetu el modelo neoliberal a escala global) surgieron reivindicaciones en torno al reconocimiento que gradualmente se impusieron sobre las reflexiones teóricas y políticas centradas en la redistribución. Para ella este desplazamiento le facilitó a las teorías feministas ampliar sus horizontes e incorporar asuntos vinculados a las diferencias (sexuales, culturales e identitarias) y, al mismo tiempo, debilitó el vínculo entre ciertos feminismos y las demandas redistributivas que promovían (y promueven) el fin de la división sexual del trabajo. Razón por la que afirma la necesidad de pensar a la justicia desde un enfoque bidimensional que articule reconocimiento y redistribución.

A nuestro juicio, siguiendo el razonamiento de Fraser, en un contexto de exacerbadas desigualdades es preciso contemplar las problemáticas culturales e identitarias sin perder de vista las propias del mundo del trabajo, sólo así podremos despuntar nuevas lecturas de la realidad que eviten interpretaciones reduccionistas y sectarias. De esta manera, a la luz del dilema redistribución-reconocimiento, nos proponemos como principal objetivo analizar, desde una perspectiva crítica e interseccional, dos políticas sociales de gran impacto en los últimos años en Argentina: la Asignación Universal Por Hijo (AUH) y el Programa Ellas Hacen (modificado por el anterior gobierno nacional encabezado por Mauricio Macri). A los fines de este trabajo creemos que el enfoque interseccional del género puede colaborar como eventual salida al aparente dilema porque permite atender a las diferentes localizaciones que los/as sujetos/as ocupan de manera simultánea. No podemos negar que nos urge visibilizar y entender cómo las múltiples desigualdades se imbrican dando lugar a situaciones de opresión particulares, según sea la experiencia vivida de cada sujeto/a y cómo estas situaciones son contempladas o no en las políticas de estado.

Para cerrar, y antes de avanzar con el análisis propuesto, consideramos ineludible decir que una primera versión de este escrito fue trabajada en el Seminario "Política Social, Derechos y Justicia Distributiva" dictado por Rosana Mazzola (FLACSO, 2019) y, posteriormente, complejizada con discusiones teóricas y lecturas enmarcadas en una investigación mayor cuyo principal objetivo busca indagar en torno a las teorías de la justicia contemporáneas ${ }^{4}$ Cabe agregar que se trata de un trabajo de análisis teórico con base en dos casos, la AUH y Ellas Hacen. En virtud del carácter predominantemente teórico, se parte de un exhaustivo proceso de revisión bibliográfica, a fin de explorar, en los casos seleccionados,

${ }^{4}$ El presente trabajo hace parte de nuestro trabajo de investigación en el marco del Proyecto "Mérito, reconocimiento y castigo en las teorías distributivas contemporáneas" de la Facultad de Derecho y Ciencias Sociales de la Universidad Nacional del Comahue y del Proyecto de Unidades Ejecutoras (PUE) "La (re)producción de las desigualdades en la Patagonia Norte. Un abordaje multidimensional" (22920180100046CO)” del IPEHCS-CONICET-UNCo. 
las posibilidades descriptivas y explicativas del debate redistribución-reconocimiento en la coyuntura actual.

\section{El dilema Redistribución-Reconocimiento}

Para comenzar debemos decir que las reivindicaciones redistributivas ponen el acento en la necesidad de una distribución justa de recursos. Esto significa que se centran en las injusticias socio-económicas, es decir, aquellas que producen explotación, marginación económica, privación de bienes materiales. Y, por otro lado, las demandas por políticas de reconocimiento buscan como fin último la "aceptación de la diferencia", haciendo referencia a injusticias culturales relacionadas a procesos de representación, interpretación y comunicación; ejemplos de ello son la dominación cultural, el irrespeto o discriminación y el no reconocimiento o reconocimiento erróneo. En la actualidad, como señalamos anteriormente, algunos/as autores/as sostienen que las luchas por el reconocimiento han extinguido a las luchas por la redistribución, dando prioridad a cuestiones simbólicas y culturales por sobre las económicas y de clase. Esta tensión entre redistribución y reconocimiento ha generado numerosos debates todavía inconclusos, entre los que se destaca el de Nancy Fraser y el afamado filósofo y sociólogo alemán Axel Honneth.

Los principales puntos de esta discusión fueron sistematizados y publicados en el libro ¿Reconocimiento o redistribución? Un debate político-filosófico (2003), a principios de los años dos mil. En términos generales, podemos decir que para ambos/as pensadores/ as la dimensión del reconocimiento es clave para reconstruir la teoría crítica de la sociedad y que coinciden en la necesidad de pensar alternativas que permitan y colaboren con la construcción de una sociedad justa. Fraser y Honneth (2003) parten de considerar a las injusticias como fenómenos complejos y, a raíz de ello, cada uno/a plantea diferentes opciones para comprender (y transformar) la realidad global contemporánea. Por su lado, Fraser relaciona con precisión -a partir de su perspectivismo dualista- a la justicia con el reconocimiento y la redistribución, mientras que Honneth le otorga al reconocimiento centralidad en todo su desarrollo teórico. Para la feminista estadounidense, la justicia requiere de la articulación entre redistribución y reconocimiento, ya que de forma aislada ambas dimensiones resultan insuficientes. Por ello, en el libro de referencia, Fraser (2003) propone una concepción bidimensional de la justicia, la cual ha complejizado con el devenir de los años.

Para graficar su planteo teórico, podemos decir que la desigualdad de género supone una estructura económica basada en la desigualdad distributiva cuyo origen es la división sexual del trabajo que distingue entre trabajo remunerado (productivo) y no remunerado (reproductivo); y, a su vez, las diferencias de estatus que se entrelazan en términos simbólicos e identitarios en una sociedad androcéntrica, dan lugar al reconocimiento erróneo de las mujeres al no considerarlas ciudadanas plenas capaces de interactuar en pie de igualdad en la vida social. Ergo, la mala distribución producto de un orden de género que estructura al capitalismo genera explotación y vulneración económica; y el no reconoci- 
miento reproduce prácticas discriminatorias y violentas fundadas en el androcentrismo. Al respecto, la politóloga feminista Line Bareiro (2010) explica que si bien es cierto que las mujeres (y otras identidades de género no hegemónicas) han alcanzado cierto nivel de reconocimiento, todavía se mantiene una distribución profundamente desigual de la riqueza y de las oportunidades, lo cual impide el ejercicio de la plena ciudadanía por parte de las mujeres. Para entender la situación de numerosos colectivos desaventajados, esta autora, introduce la noción de ciudadanía restringida, a la cual define como "la dificultad o imposibilidad de ejercer derechos por parte de personas que declarativamente son ciudadanas con todos los derechos que implica el estatus" (2010, p. 24).

En este sentido, si retomamos los aportes de Adelantado et al (2000) podemos decir que es posible tener una concepción dual de la política social, en tanto constitutiva y moduladora de las desigualdades sociales. Esto significa que la política social no se reduce a una mera intervención compensatoria de los efectos negativos del mercado (2000, p. 17). Pues, las políticas públicas muchas veces atacan las consecuencias desiguales del modo de producción capitalista pero no apuntan a sus causas y pilares fundamentales. De hecho, como indica Claudia Anzorena (2014), en la práctica estatal las políticas sociales son pensadas como "políticas sexualmente neutras" cuya finalidad es atender a la situación de pobreza y marginalidad de ciertos sectores; mientras que se consideran "políticas de género" a aquellas dirigidas exclusivamente a las mujeres (2014, p. 34). De esta manera se consolida de manera operativa una visión y un abordaje departamentalizado de las desigualdades. Sobre este punto, Anzorena redobla su crítica y agrega: "[e]sta ilusión de compartimentos estancos exime a la burocracia estatal y a sus funcionarios/as de hacerse cargo de que clase, racialización y género están fuertemente imbricados" (2014, p. 34). Por esta razón, insistimos en la importancia de incorporar operativamente una perspectiva basada en la interseccionalidad del género, ya que esta mirada constituye una apuesta teórica con gran potencial político.

En este sentido, cabe mencionar que la interseccionalidad como herramienta -teórica y metodológica- tuvo su origen en el pensamiento y la lucha de feministas negras, ya que surgió para explicar y entender las experiencias de las mujeres afrodescendientes en Estados Unidos. Se alude a la jurista Kimerlé Williams Crenshaw como la responsable de acuñar dicho término hacia la década de los ochenta. Desde entonces, la interseccionalidad sirvió (y sirve) como un instrumento útil para interpretar el racismo y el sexismo como fenómenos que operan mediante dispositivos afines. En otras palabras, permite comprender la convergencia de las diferentes formas de opresión/desigualdades padecidas por las mujeres, ligadas al sexismo, clasismo y racismo (Zambrini, 2015). Es decir, la interseccionalidad muestra la importancia de desarrollar análisis que incluyan las diversas (y multidimensionales) estructuras de poder. También es preciso advertir que, de un tiempo a esta parte, la interseccionalidad está en boga y es parte del repertorio de los discursos políticamente correctos, lo cual supone cierto riesgo como, por caso, volverlo un concepto estéril sin posibilidad de materializarse en prácticas concretas orientadas a la equidad.

La selección de los casos que presentamos y analizamos en este documento ha sido intencional, puesto que nos interesa exponer cómo en las políticas sociales se tiende a 
mostrar que las desigualdades de género son independientes de las económicas y ajenas a otras formas de injusticia cultural. Por ello escogimos dos políticas que de manera directa y/o indirecta se relacionan con "las mujeres". Nos importa aclarar que si bien la AUH es una política orientada a las infancias, son en general las mujeres-madres las responsables directas de sostener el beneficio (Genolet, Carmody, Lauphan y Guerreira, 2016; Bonavitta, 2018; Micha, 2019); mientras que el programa Ellas Hacen ha sido destinado a mujeres que cobran la AUH y/o víctimas de violencia (Fernández y Pieruzzini Cid, 2017; Arcidiácono y Bermúdez, 2017). Creemos que focalizarnos en la concepción del sujeto mujer(es) que se aplica en ambos casos puede abrirnos un singular umbral que nos permita abonar una mirada crítica y que facilite nuevos análisis. Seguidamente ahondamos en ambas políticas a partir de esta clave e intentamos echar luz sobre las fortalezas y debilidades de ambas propuestas.

\section{Políticas Sociales en Argentina}

Los debates distributivos suponen posiciones sobre lo "aceptable o no de las diferencias económicas entre personas” (Gasparini, Cicowiez y Escudero, 2014). En qué medida es aceptable la desigualdad económica y qué hacer frente a ella nos conduce necesariamente, como ya dijimos, a discusiones sobre la justicia. En este punto aparece la equidad como concepto clave para evaluar si una situación es justa o no, si las diferencias deben ser aceptadas o combatidas y por qué. Lo cierto es que los diversos enfoques existentes difieren, en general, en la identificación de la variable que consideran importante igualar para alcanzar una situación equitativa (Gasparini et al., 2014), a saber: igualdad de resultados; igualdad de oportunidades ${ }^{5}$. Ciertamente, como es de común conocimiento, la región de América Latina y El Caribe es una de las más desiguales del mundo. La medición de la desigualdad en términos de ingreso es, para algunos autores/as, fundamental al momento de evaluar el bienestar de la población y, claramente, para la eventual puesta en funcionamiento de políticas públicas. Vale agregar que en América Latina se ha impuesto con notoriedad la métrica basada en los ingresos como variable y los consiguientes datos producidos a partir de encuestas de hogares (Jiménez y Amarante, 2015).

Las problemáticas vinculadas con la desigualdad económica y, especialmente con la pobreza, se imponen con notoriedad en las agendas de esta región. Ciertamente la inversión social es central para avanzar en la justicia distributiva y lograr una ampliación de derechos. Considerando que el mercado nunca garantizará por sí mismo un reparto de la riqueza de manera equitativa, se requiere de una activa participación del Estado para promover dicho reparto y ampliar oportunidades a favor de los/as más vulnerables o desaventajados/as. Por esta razón, las políticas orientadas a dichos sectores suelen colocar

\footnotetext{
${ }^{5}$ La igualdad de oportunidades tiene como finalidad otorgar a todos/as los/as individuos/as las mismas posibilidades de ocupar en la estructura social una posición más favorable. En cambio, la igualdad de posiciones busca reducir las brechas entre los/as individuos/as que ocupan diferentes posiciones en la estructura social, es decir, no apunta a eliminar dichas desigualdades.
} 
su atención en demandas por redistribución de bienestar social. Esta tendencia puede generar, al mismo tiempo, la reproducción de estigmas y el reforzamiento de estereotipos y roles que tienen como efecto el reconocimiento erróneo de dichos grupos. En este sentido nos importa destacar que pensamos a la estigmatización como parte de una construcción social relacional, en tanto representación colectiva, que colabora con la construcción de fronteras morales (Bayón, 2020).

A través de la estigmatización de sectores subalternos no sólo se etiqueta a las personas sino que se refuerzan estereotipos negativos que, a su vez, suponen una pérdida de estatus. La figura de la mamá luchona ${ }^{6}$ es una síntesis de la estigmatización que padecen las mujeres que de forma indirecta son beneficiarias de la AUH. El estigma es una herramienta de poder en tanto tiene la posibilidad de excluir, dejar por fuera o por abajo a ciertos grupos, es decir, cumple la función de limitar el acceso a oportunidades. La clase se espacializa (identificándose barrios, villas, asentamientos, tomas) y se encarna en personas con determinada apariencia, de este modo no sólo se segrega a ciertos grupos sino que también se los etiqueta y criminaliza (Bayón, 2020). Alcanza con observar los orígenes de la $\mathrm{AUH}^{7}$ para comprender la manera en la que operan estigmas y estereotipos sobre las familias beneficiarias y cómo las mujeres de sectores empobrecidos se llevan la peor parte: "se embarazan para cobrar la AUH". Frases de este tenor se multiplican en los medios hegemónicos desde que se inició con esta política y ha estado en boca de funcionarios/as de distintos colores del arco político nacional.

En Argentina los hogares nucleares y con jefatura masculina continúan siendo los predominantes, aunque, los hogares unipersonales y con jefatura de hogar femenina crecieron con notoriedad, pasando del $15 \%$ al $17.7 \%$ y del $27.6 \%$ al $34.1 \%$ respectivamente (Mazzola, 2016, p. 64). En la coyuntura actual, gran parte de los/as trabajadores/as precarizados/as y desocupados/as son mujeres. Por tanto, es lógico que las principales beneficiarias de la AUH sean familias encabezadas por mujeres madres en situación de vulnerabilidad económica, lo que las transforma en responsables de las contraprestaciones que condicionan a dicha política. Estas contraprestaciones suponen tareas de cuidado de las infancias, las cuales históricamente descansan sobre las mujeres. Aquí observamos cómo se refuerzan roles estereotipados que operan negativamente sobre la vida y las oportunidades de las mujeres.

No es casual, entonces, que las economistas feministas sostengan que las tareas de cuidado son la clave de la desigualdad económica. De este modo, observamos la interrelación existente entre la desigual distribución y el reconocimiento erróneo que padecen las mujeres. Los estereotipos sexistas no sólo motorizan, multiplican y legitiman la discri-

\footnotetext{
${ }^{6}$ Se denomina "mamá luchona" a las madres solteras que enfrentan la crianza de manera individual. En Argentina, especialmente en redes sociales, se señala peyorativamente a las madres solteras pobres. De un tiempo a esta parte, ha crecido con notoriedad la estigmatización de aquellas mujeres de sectores populares a quienes se les condena por publicar fotografías en redes de sus hijos/ (a quienes presentan como "bendiciones de dios"), se cuestiona su vestimenta e incluso sus nombres de pila son motivo de burla. La población objetivo de tal discriminación son las mujeres madres solteras que residen en barrios urbanos marginales, villas o tomas.

${ }^{7}$ Tal como veremos más adelante la AUH, al momento de su creación, tenía como finalidad la ampliación especial del sistema de seguridad social para los/as hijos/as de los/as trabajadores/as del sector informal, de las trabajadoras domésticas y para los hijos/as de los/as desocupados/as (Maneiro, 2017, p. 616).
} 
minación social de las mujeres sino que también sirven como obstáculos para el acceso a derechos. Así, vemos como la tríada mujer-madre-cuidadora no sólo obtura el ingreso de las mujeres al mercado laboral sino que también colabora con la estigmatización que viene ligada al régimen neoliberal. A continuación abordamos con especial atención la AUH, puesto que entendemos que es imperioso pensar y re-pensar el lugar que ocupan las mujeres en las políticas sociales. Creemos que sostener miradas estereotipadas, atenta contra el principio de igualdad sobre el cual se erigen los derechos humanos. En palabras de Pautassi, Arcidiácomo y Straschnoy:

las políticas sociales muestran límites concretos, no sólo en términos de impacto redistributivo, sino que claramente desnudan cómo este principio ha sido incluido de manera retórica, asociado al imperativo de promover marcos de inclusión social, pero en los hechos, poco se ha avanzado en el diseño de sistemas integrales respetuosos del conjunto de derechos humanos, y en muchos casos se ha discriminado. (2013, p. 64)

\section{La Asignación Universal por Hijo}

La AUH se inició en 2009 y, desde entonces, está destinada a las familias en situación de vulnerabilidad (personas desocupadas, que trabajan en el circuito informal o cuyos ingresos son menores al salario mínimo, vital y móvil) por cada hijo/a menor de 18 años (o sin límite de edad en caso de tener una discapacidad), y hasta un máximo de 5 hijos/as por grupo familiar. Durante el año 2011 se incorporó a las mujeres embarazadas (AUE), a partir de las 12 semanas de gestación. Según datos de ANSES (2012) el 94,4\% son mujeres madres o tutoras quienes perciben la asignación. A criterio de Laura Pautassi (2013), a partir de estos datos se observa cierta feminización de la AUH, la cual promueve "nuevas" vulnerabilidades como el hecho de ser "madre y pobre" en desmedro de la autonomía de las mujeres. Asimismo, esto da cuenta de la sobrecarga de tareas que ocupan a las mujeres vistas como únicas responsables de los cuidados y, en última instancia, del éxito o fracaso del programa.

A todo esto, no podemos perder de vista que la AUH es un PTC punitivo, ya que supone la obligación de que los/as niños/as asistan al colegio, tengan las vacunas al día y se realicen controles médicos oportunos. Consideramos que se trata de un PTC con características "punitivas" porque el incumplimiento es causal de recorte y/o pérdida del beneficio. Aquí otro nodo problemático de esta política, pues a nuestro parecer los derechos no deben estar condicionados y mucho menos reproducir estereotipos y roles sociales que colaboran con la subordinación de las mujeres. La corresponsabilidad familiar y parental es menester al momento de pensar en las políticas de protección de las infancias, de lo contrario se perpetúa un orden de géneros patriarcal y androcéntrico que oprime a las mujeres y las sujetas a situaciones de desigualdad, confinándolas al espacio privado e imposibilitando su inserción en el mercado laboral.

Según Claudia Anzorena (2015), a pesar de que las mujeres no son las titulares del derecho en la AUH vemos cierta exaltación de "la madre" y su "papel heroico", lo cual no 
responde a una valoración de las mujeres como ciudadanas sino a concepciones que portan los/as funcionarios/as, cargadas de supuestos generizados. Estos supuestos exacerban tanto los valores morales atribuidos a lo femenino y a la maternidad, como la idea de que las mujeres están espontáneamente más inclinadas al trabajo de cuidado de los/as otros/as (Anzorena, 2015, p. 112). Ser una "mamá luchona" es presentado como una virtud y, al mismo tiempo, supone un estigma social muy potente. No son pocas las políticas que en busca de formas de redistribución de bienestar social, generan también efectos de reconocimiento erróneo (discriminación y estigmatización). Tal como indicamos anteriormente este es un caso que nos permite visualizar con claridad cómo se estigmatiza a ciertos sectores sociales y se afianzan estereotipos negativos, por caso "ser un parásito del estado", "todo/as vagos/as que no quieren trabajar", "negros/as choriplaneros". El problema surge, siguiendo el razonamiento de Fraser (2008), cuando las políticas de redistribución tienen injerencias en las identidades de los/as actores/as sociales y en su posicionamiento económico pero no son acompañadas por cambios culturales que atiendan a la injusticia que supone la discriminación o reconocimiento erróneo. El sexismo, la estigmatización y racialización que padecen estos sectores nos permite visualizar la imbricación existente entre el género, la clase y la raza (en tanto patrón de poder).

En contextos de ajuste estructural, vemos cómo mientras el Estado exige a las mujeres que cumplan con las tareas de reproducción y cuidado se ha corrido de su papel de garante último de los derechos de las infancias, ejemplo de ello es la falta de inversión que hubo en los últimos años en materia de salud y educación pública. Así, no sólo se omiten las contingencias en el núcleo familiar sino que también se agrega el déficit de infraestructura pública de cuidado (Pautassi et al., 2013). Aquí se observa la ausencia de una perspectiva interseccional del género; el Estado exige a "las mujeres" ignorando por completo las múltiples experiencias y situaciones particulares y replica roles estereotipados que perpetúan la subordinación de género. De este modo los pisos pegajosos y las escaleras rotas -que denuncian las economistas feministas- se multiplican y continúan excluyendo a las mujeres del mercado laboral y negando las múltiples formas de dominación que las oprimen. Entonces, como sostiene Mazzola (2015), si bien hubo importantes avances en materia de seguridad social, a partir de la AUH y AUE, todavía queda mucho por fortalecer y mejorar sobre todo en pos de garantizar los derechos de las mujeres madres y trabajadoras.

\section{Ellas Hacen}

El Programa Ellas Hacen se originó -en 2013- como un incentivo económico destinado a mujeres madres receptoras de la AUH con una contrapartida de trabajo, capacitación y finalización de estudios (Anzorena, 2015). Ellas Hacen priorizaba a aquellas mujeres en situación de mayor vulnerabilidad: madres con tres o más hijos/as, con hijos/ as con discapacidad y/o las víctimas de violencia de género. A priori podemos señalar que el programa surgió como una forma de impulsar el "trabajo digno", por lo que se encuadró dentro del programa nacional "Ingreso social con Trabajo-Argentina Trabaja”. Uno de los 
principales objetivos consistía en "construir ciudadanía" en villas y barrios emergentes, sin embargo, en la práctica no logró romper la lógica estigmatizadora que replica estereotipos negativos como el de "mujer-madre-cuidadora", pensado como núcleo duro de la vulnerabilidad. Si bien el programa promovía la conformación de espacios cooperativos de trabajo, como una forma de trascender las fronteras de lo doméstico no se supera el sesgo de género en términos de cuidado: ¿quién cuida mientras ellas hacen? El Estado exige a las mujeres tareas de cuidado y, al mismo tiempo, busca "empoderarlas" e insertarlas en el mercado laboral sin considerar las asimetrías reales que existen en nuestra sociedad.

También debemos considerar que muchas veces en busca de promover el afamado "empoderamiento", se impulsan políticas cuyas destinatarias son las mujeres-madres excluyéndose a otras identidades de género no hegemónicas (mujeres trans y travestis), las mujeres madres con hijos/as mayores de edad e incluso a las mujeres no madres. En este punto no es menor señalar la forma en la que sistemáticamente los organismos estatales, a través de sus políticas, han omitido e ignorado el masivo reclamo por la autonomía y el control de los cuerpos gestantes que encabezan las organizaciones feministas. De hecho, actualmente, en medio de acalorados debates por la Ley de Interrupción Voluntaria del Embarazo, se divisa como muchos/as funcionarios/as prefieren hacer referencia a la necesidad de avanzar con políticas de protección destinadas a las mujeres-madres en lugar de garantizar políticas de salud, lo cual corre el eje de la discusión y niega e invisibiliza los derechos sexuales y reproductivos de las mujeres y otros cuerpos gestantes.

El programa Ellas Hacen se ha visto modificado por el saliente gobierno nacional, en la actualidad se han fusionado varios programas dando lugar a "Hacemos futuro", plan orientado a la finalización de la educación formal obligatoria y a la formación integral. Sin duda, el potencial no cuantificable de Ellas Hacen radica en la generación de vínculos y redes entre mujeres, en la construcción de comunidad y en la re-significación de la espacialidad cotidiana (el barrio, la toma, el asentamiento, la villa) como territorio libre de violencias. Y si bien estos son avances en materia de género, no podemos negar que la normativa vigente reconoce distintos tipos y modalidades de violencia contra las mujeres, dentro de ellas la violencia simbólica. Esta forma de violencia refiere directamente a patrones estereotipados que sirven como obstáculo para la vida plena de las mujeres. Por tanto, creemos que seguir colocando a las mujeres en el rol de cuidadoras y únicas responsables de tareas propias del ámbito de lo privado-doméstico es una forma de estereotipación que obtura las oportunidades de una vida justa y equitativa.

En la coyuntura que nos toca, la urgencia que supone la pobreza hace que las cuestiones distributivas opaquen las demandas por reconocimiento que encarnan ciertos sectores que padecen múltiples formas de discriminación. Y, al mismo tiempo, las reivindicaciones identitarias y culturales de ciertos grupos y colectivos han perdido de vista las desigualdades estructurales que afectan a gran parte de la población de toda la región latinoamericana. Los feminismos han contribuido notoriamente al denunciar la necesidad de atender de manera integral la situación desigual que padecen las mujeres, sin que esto suponga un sesgo culturalista que vuelva a ignorar (como ocurrió durante la década de los noventa) la cuestión económica estructural y viceversa. Por esta razón, compartimos las 
apreciaciones teóricas de Fraser al asegurar que es urgente pensar estrategias que articulen políticas de reconocimiento con políticas de re-distribución a fin de alcanzar la justicia de género y consolidar sociedades más justas y equitativas.

\section{Notas finales}

La profunda crisis que azotó a Argentina iniciados los años dos mil obligó al Estado a tomar cartas en el asunto, impulsándolo a generar políticas sociales de inclusión destinadas a generar transferencias monetarias a los sectores desaventajados. A pesar de que con el tiempo se alcanzó cierto crecimiento económico y una paulatina recuperación a nivel de empleo, importantes sectores sociales continuaron en situación de vulnerabilidad, por lo que se crearon políticas como la AUH y la AUE. Lo novedoso de ambos casos es que por primera vez se incluyó a trabajadores/as desocupados/as o empleados/as en trabajos informales. Además, se priorizó a la "madre" como titular del derecho (Guerrero et al, s.f). Y, justamente, este es uno de los puntos que buscamos problematizar en el presente trabajo. En las dos políticas seleccionadas, AUH y Ellas Hacen, se puede evidenciar un reforzamiento de roles estereotipados que recluyen a las mujeres al espacio de lo doméstico, pues ambas hacen énfasis en la mujer-madre-cuidadora responsable -directa y exclusivade las tareas reproductivas. Entender a las mujeres como destinatarias de un beneficio monetario no supone, necesariamente, que se incorpore un enfoque de género, ni que exista un compromiso en la promoción de los derechos de las mujeres. Y, claramente, en estos casos observamos beneficios derivados y supeditados a la condición de "madre".

La asimetría en la distribución del trabajo doméstico y de las tareas de cuidado es una de las principales fuentes de desigualdad de género y, como señala Mercedes D'Alessandro (2017), trasciende la brecha salarial. Las mujeres dedican mucho tiempo a estas actividades/tareas por lo que cuentan con menos posibilidades/oportunidades de ingresar al mercado laboral, finalizar estudios e incluso cuentan con menos tiempo de ocio que los varones. La doble o triple jornada laboral a la que las mujeres se ven expuestas se conjuga con la precarización y flexibilización laboral propia del neoliberalismo tardío. Si bien es cierto que en los últimos años las mujeres han logrado mayor inserción en el mercado de trabajo, esto no se ha traducido en el reparto de las tareas de cuidado o en la co-responsabilidad parental ${ }^{8}$. La trillada frase "mi marido me ayuda con la casa" da cuenta de la vigencia de ciertos roles y estereotipos de género, pues se trata de construcciones sociales (relacionales) que operan de manera transparente, se naturalizan y se hacen carne en la cotidianeidad de cientos y miles de mujeres.

\footnotetext{
${ }^{8}$ A fin de poner en valor "buenas prácticas", es preciso indicar que en ciertos países se ha avanzado en políticas públicas que promueven la co-responsabilidad parental, por ejemplo en Finlandia se igualaron los permisos de paternidad y maternidad eliminándose las referencias al género (ahora se consideran "permisos familiares"). En el caso de Argentina, las licencias por paternidad/maternidad varían según el sector y la provincia. La licencia por paternidad para empleados del sector privado suele ser de 2 días corridos, una de las más cortas de toda América Latina. Cabe señalar que en el caso de Buenos Aires (CABA) se ha observado una ampliación de derechos con el nuevo régimen de licencias familiares aprobado en 2018.
} 
La AUH en tanto PTC exige a las mujeres que los/as hijos/as asistan a la escuela, tengan controles médicos periódicos, etc. Todo esto sin tener en cuenta las múltiples realidades y situaciones particulares de estas mujeres: no es lo mismo vivir en un barrio urbano marginal en CABA que en una comunidad rural del Gran Chaco argentino. No podemos negar que la AUH significó y significa un gran avance en materia de protección ampliada de derechos de las infancias (Mazzola, 2015), pese a ello consideramos menester incorporar de manera transversal la perspectiva de género. A nuestro criterio todavía tenemos un gran desafío por delante: dejar atrás las generalidades que sirven de cimientos de políticas sociales y comenzar a pensar en términos interseccionales las imbricaciones entre de género, raza-etnia, clase, discapacidad, etc. Transversalizar la perspectiva interseccional de género en los organismos del estado debe ser una meta a corto y mediano plazo, solo de este modo se podrá superar la mirada reduccionista que se ha impuesto a lo largo del tiempo. Todavía la mayoría de las políticas que se enuncian como "políticas de género" atienden pura y exclusivamente las demandas de sectores (cis)género en tanto "mujeres" y esto no es suficiente para erradicar la violencia y desigualdad que padecen numerosos grupos desaventajados de nuestra sociedad.

En esta misma dirección abordamos el programa Ellas Hacen, el cual fue creado a partir de la necesidad real de generar mayor inclusión de las mujeres que se encontraban en situaciones de precariedad. Nos importa reiterar que las jefaturas femeninas de hogar en Argentina crecieron a la par del proceso de feminización de la pobreza. La contraprestación solicitada en el caso del programa Ellas Hacen, consistía en la finalización de los estudios (FINES) y la conformación de cooperativas de trabajo. Las mujeres destinatarias eran "jefas" de hogares monoparentales y/o víctimas de violencia, esto coloca nuevamente en el centro de la escena a las mujeres-madres y con ello al rol maternal como mandato social dominante. Según Anzorena: "el Estado tiene una relación ambivalente con las mujeres: a veces las considera pobres pertenecientes a grupos vulnerables, a veces madres responsables de la supervivencia de otros/as, a veces ciudadanas con (ciertos) derechos; pero escasas veces se combinan" (2015, p. 99). Además, si bien el mencionado programa se proponía, como lineamiento general, que las mujeres puedan desnaturalizar la opresión por razón de género y salir de situaciones de violencia, lo cierto es que era condición necesaria para ingresar al programa ser "madre", como hemos dicho, esto no toma en cuenta la lucha por la autonomía de los cuerpos e invisibiliza a numerosas identidades que no se ajustan a la heteronorma.

El cuidado de los niños/as mientras las madres realizaban las capacitaciones o trabajaban en las cooperativas continuó siendo un problema del ámbito de lo privado y responsabilidad de las mujeres madres. Por tanto, ambos casos, la AUH y Ellas Hacen, nos muestran la urgencia de elaborar políticas de afirmación que se propongan corregir las desigualdades distributivas a corto plazo sin que se pierda de vista la necesidad imperiosa de aplicar políticas de transformación orientadas a desarticular relaciones desiguales de poder que generan el irrespeto o reconocimiento erróneo de las mujeres en la sociedad. Las injusticias que suponen las desigualdades de género requieren soluciones tanto económicas como culturales e identitarias. Estaremos frente a la imposibilidad de alcanzar 
la justicia de género si no logramos la articulación de políticas de redistribución y reconocimiento, ya que como señala Fraser (2003) su distinción una cuestión exclusivamente analítica.

Esto será factible si se incorpora una perspectiva interseccional del género que atienda de manera integral a las demandas por políticas de reconocimiento y redistribución que tienen las mujeres (y otras identidades de género no hegemónicas). No sólo se hace urgente establecer diálogos o nexos entre el reconocimiento y la cuestión distributiva sino que es preciso generar espacios de diálogo y la participación política de las mujeres, lo que Fraser considera como tercera dimensión de la justicia: la representación. La participación social, política y comunitaria es primordial si nos posicionamos desde una perspectiva o enfoque de derechos que considere a los/as sujetos/as destinatarios/ as como titulares de derechos, capaces de participar democráticamente en la elaboración y ejecución de las políticas de estado. Por tanto, transversalizar la perspectiva de género desde una mirada interseccional debe ser un compromiso activo y colectivo que evite cristalizar enfoques y que favorezca el diálogo/debate político en pos de una sociedad justa, equitativa y democrática.

\section{Referencias bibliográficas}

Adelantado, J., Noguera, J. y Rambla X. (2000). “El marco de análisis: las relaciones complejas entre estructura social y políticas sociales". En Adelantado, J. (Coord.) Cambios en el Estado de Bienestar. Políticas sociales y desigualdades en España (pp. 23-62). Editorial Icari.

Anzorena, C. (2014). Aportes conceptuales y prácticos de los feminismos para el estudio del Estado y las políticas públicas. Revista de Trabajo Social Plaza Pública, 7 (11), pp. 17-41.

AnzorenA, C. (2015). ¿Qué implica la protección social para las mujeres? Un análisis feminista de las políticas sociales y de igualdad en la Argentina. OXÍMORA. Revista Internacional de Ética y Política, 7, 98-118.

Arcidiácono, P. y Bermúdez, A. (2018). “Ellas hacen”. Programas sociales y exigencias a las mujeres en Argentina. Revista Estudios Feministas, Florianópolos, 26 (2), 1-16. http://dx.doi.org/10.1590/1806-9584-2018v26n245297

BAREIRo, L. (2010). Ciudadanía, democracia y Estado en plena transformación. Anuario de Derechos Humanos. Recuperado de www.anuariocdh.uchile.cl

BAYón, M. (2020). Hacia una sociología del estigma: desclasificando los sectores populares. En Seminario General-Modalidad Virtual llevado a cabo por el IDAES-UNSAM, Buenos Aires.

BonavitTa, P. (2018). Asignación universal por hijo y los roles de género. Revista Punto Género, 8, 4-19. doi:10.5354/0719-0417.2017.48398

D'Alessandro, M. (2017). Economía feminista. Cómo construir una sociedad igualitaria (sin perder el glamour). Buenos Aires: Editorial Sudamericana. 
ESPING ANDERSEN, G. (2000). Fundamentos sociales de las economías postindustriales. Editorial Ariel.

FERnÁndez, M. y PieruZzini Cid, R. (2017). "Ellas hacen": análisis de una política pública desde una perspectiva de género. Voces emergentes, 1, 46-55. http://sedici.unlp.edu. ar/handle/10915/63755

Fraser, N. y Honneth, A. (2003). ¿Reconocimiento o redistribución? Un debate políticofilosófico. Morata.

FraSER, N. (2008). "La justicia social en la era de la política de la identidad: redistribución, reconocimiento y participación”. Revista de trabajo, 4(6), 83-99.

FRASER, N. (2012). "La política feminista en la era del reconocimiento: un enfoque bidimensional de la justicia de género". ARENAL, 19(2), 267-286.

Gargarella, Roberto (1999): Las teorías de la justicia después de Rawls. Paidós.

Gasparini, L., Cicowiez M. y Escudero, W. (2014). Pobreza y Desigualdad en América Latina: Conceptos, Herramientas y Aplicaciones. Editorial Temas.

Genolet, A.; Carmody, C.; Lauphan, W. y Guerriera, L. (2016). Avances y desafíos de la AUH. Una mirada desde el género y la perspectiva de derechos. Ciencia, Docencia y Tecnología Suplemento, 6 (6), 440-455.

JimÉNEZ, J.P. y AMARANTE, V. (2015). Desigualdad, concentración y rentas altas en América Latina. En Jiménez, J. (Ed.) (2015) Desigualdad, concentración del ingreso y tributación sobre las altas rentas en América Latina (pp. 13-27). CEPAL.

MANEIRo, M. (2017). Representaciones sociales sobre la Asignación Universal por Hijo de los sectores populares urbanos periféricos. Trabajo y Sociedad, 29, 611-629.

Mazzola, R. (2015). Nuevo Paradigma. La Asignación Universal por Hijo en la Argentina. Editorial Prometeo.

Mazzola, R. (2016). "Primera infancia en Argentina Relaciones entre (des) igualdad, familias y políticas de bienestar". Revista Estado y Políticas Públicas (6), 59-95. https:// revistaeypp.flacso.org.ar/revista/numero-6_19

Mazzola, R. (22 de mayo de 2016). Infancias y mujeres vulnerables. Página 12. https:// www.pagina12.com.ar/diario/suplementos/cash/17-9340-2016-05-22.html

MichA, A. (2019). Usos y administración de la Asignación Universal por Hijo (AUH) entre el "deber ser" y la autonomía económica de las mujeres. Trabajo y Sociedad, 32, 359-386.

Pautassi, L; Arcidiácomo, P. y Straschnoy, M. (2013). Asignación Universal por Hijo para la Protección Social en Argentina. Entre la satisfacción de necesidades y el reconocimiento de derechos. Santiago de Chile: Naciones Unidas.

PAUTASSI, L. (2013). La igualdad en las políticas sociales. Retóricas presentes, obligaciones pendientes. Revista de la Facultad de Ciencias Sociales-UBA, 84, pp. 64-69.

RAWLs, J. (2000 [1971]). Teoría de la Justicia. México: FCE.

WAlzer, M. (1993) Las esferas de la justicia. Una defensa del pluralismo y la igualdad. Fondo de Cultura Económica.

ZAMBRINI, L. (2015). Diálogos entre el feminismo postestructuralista y la teoría de la interseccionalidad de los géneros. Revista Punto Género, (4), pp. 43-54. doi:10.5354/07190417.2014.36408. 